\title{
Antidepressant use in pregnancy and severe cardiac malformations: Danish register-based study
}

\author{
Line Kolding ${ }^{1}$, Vera Ehrenstein ${ }^{1}$, Lars Pedersen ${ }^{1}$, Puk Sandager ${ }^{1}$, Olav Petersen ${ }^{2}$, Niels \\ Uldbjerg $^{1}$, and Lars Pedersen ${ }^{1}$ \\ ${ }^{1}$ Aarhus University Hospital \\ ${ }^{2}$ Copenhagen University Hospital Rigshospitalet
}

October 12,2020

\begin{abstract}
Objective Studies restricted to live births may underestimate severe teratogenic effects. We address the limitation by including data from both prenatal and postnatal diagnoses of cardiac malformations. Design Register-based study. Setting Denmark. Population 364,012 singleton pregnancies from 2007 to 2014. Methods We used data from five nationwide registries. Exposure to antidepressants was measured using redeemed prescriptions. Main Outcome Measures Pregnancies with cardiac malformations that end in miscarriage, termination, stillbirth, postnatal death or cardiac surgery $<1$ year of birth were classified as severe cardiac malformations (SCM). Propensity scores with adjusted prevalence ratios (PRs) were calculated. Results SCM were reported in 972 / 364012 pregnancies overall and in 16 / 4105 exposed. PRs for SCM were 1.09 (95\%CI: 0.52-2.30) for selective serotonin reuptake inhibitors (SSRIs) and 2.13 (95\%CI: 0.89-5.13) for venlafaxine. Among the venlafaxine-exposed pregnancies, there was a cluster of hypoplastic left heart syndromes (HLHS) (crude PR 17.4 (95\%CI: 6.41-47.2)) of which none ended in a live birth. For HLHS, the absolute risk increase was 4.4 per 1000, the number needed to harm (NNH) was 225. PRs for cardiac malformations not classified as SCM were 1.38 (95\%CI: 1.00-1.92) for SSRIs, and 1.73 (95\%CI: 1.08-2.77) for venlafaxine. Conclusions Pregnancy exposure to venlafaxine, but not SSRIs, is associated with an increased risk of SCM but with a low absolute risk. Potential mechanisms include direct effects or confounding by indication. Venlafaxine exposure is a marker for risk pregnancies for which fetal echocardiography may be considered. Keywords pregnancy, antidepressants, SSRI, venlafaxine, prenatal ultrasound, selection bias
\end{abstract}

\section{Hosted file}

AD Manuscript_BJOG_final1.pdf available at https://authorea.com/users/366624/articles/486315antidepressant-use-in-pregnancy-and-severe-cardiac-malformations-danish-register-basedstudy 